\title{
Detecting Green Mold Pathogens on Lemons Using Hyperspectral Images
}

\author{
Yuriy Vashpanov ${ }^{1} \oplus$, Gwanghee Heo ${ }^{1,2} \oplus$, Yongsuk Kim ${ }^{1,3}$, Tetiana Venkel ${ }^{4}$ and \\ Jung-Young Son $1, *$ (D) \\ 1 Public Safety Research Center, Konyang University, Nonsan 32992, Korea; \\ yuriy.vashpanov@gmail.com (Y.V.); heo@konyang.ac.kr (G.H.); yongsuk@konyang.ac.kr (Y.K.) \\ 2 Department of Civil Engineering, Konyang University, Nonsan 32992, Korea \\ 3 Department of Bio-IT Engineering, Konyang University, Nonsan 32992, Korea \\ 4 Linguistics for Sciences Department, Chernivtsi University, 58012 Chernivtsi, Ukraine; t.venkel@chnu.edu.ua \\ * Correspondence: jyson@konyang.ac.kr; Tel.: +82-41-730-5636
}

Received: 19 December 2019; Accepted: 6 February 2020; Published: 11 February 2020

check for updates

\begin{abstract}
Hyperspectral images in the spectral wavelength range of $500 \mathrm{~nm}$ to $650 \mathrm{~nm}$ are used to detect green mold pathogens, which are parasitic on the surface of lemons. The images reveal that the spectral range of $500 \mathrm{~nm}$ to $560 \mathrm{~nm}$ is appropriate for detecting the early stage of development of the pathogen in the lemon, because the spectral intensity is proportional to the infection degree. Within the range, it was found that the dominant spectral wavelengths of the fresh lemon and the green mold pathogen are $580 \mathrm{~nm}$ and $550 \mathrm{~nm}$, respectively, with the $550 \mathrm{~nm}$ being the most sensitive in detecting the pathogen with spectral imaging. The spectral intensity ratio of the infected lemon to the fresh one in the spectral range of $500 \mathrm{~nm}$ to $560 \mathrm{~nm}$ increases with the increasing degree of the infection. Therefore, the ratio can be used to effectively estimate the degree of lemons infecting by the green mold pathogens. It also shows that the sudden decrease of the spectral intensity corresponding to the dominant spectral wavelength of the fresh lemon, together with the neighboring spectral wavelengths can be used to classify fresh and contaminated lemons. The spectral intensity ratio of discriminating the fresh lemon from the infected one is calculated as 1.15 .
\end{abstract}

Keywords: healthy and infected lemons; Hyperspectral image; Penicillium digitatum pathogen; lemon skin; dominant spectral wavelength; spectral intensity ratio

\section{Introduction}

As the volume of global production of fruits increases, the use of a spectroscopic method for the purposes of monitoring surface condition and controlling the quality of the fruits in food and agricultural industries is continuously increasing, especially in determining the ripening status [1]. This is because the method is rapid, simple, and accurate for the above-mentioned purposes, as the visual colors of the fruit skin are directly related to the wavelengths of the light reflected from objects' surfaces, especially in the visible and near infrared spectral ranges. In addition, the spectroscopic method is noninvasive. As the most fruits are soft, any form of physical forces affecting their skin, such as collisions among them and shocks from outside causes bruises and scars of the skin. The bruises and scars can induce changes in the skin color of the fruits. Moreover, the juicy components emitted to the skin in the process of forming the bruises and scars can cause various diseases in fruits. These diseases are causing further changes in the skin color of fruits. Therefore, the noninvasive method is preferable for the inspection of fruits.

Among the diseases, fungal infections induce such serious problems as asthma, cancer, and other diseases to human health [2-6]. As the fungi spores widely spread in the environment are gradually 
accumulated in the human body, the long-term exposure to them can induce damages to internal organs. Therefore, they are a significant threat to human health. In fact, it is known that more than a billion people suffer from the diseases, and more than 1.5 million people die each year by the diseases [7]. Among the fungi, the pathogen Penicillium digitatum (green mold) is the fungus that is dominantly found in the skins of citrus fruits, especially in lemons, and is very abundant in our environment $[8,9]$. The diseases in fruits outbreak when the biological pathogens infiltrate the fruits' surfaces. As a consequence, the fruits' skins change their colors, and other healthy fruits near the infected ones are contaminated. Thus, the infected fruits should be separated from the healthy ones. For this purpose, a quick and accurate method of identifying the infected fruits, i.e., inspecting the large amount of fruits in real-time and quantifying the degree of the infections should be devised. In this regard, the spectral imaging, which photographs an object or scene with the use of a spectral filter, has been recognized as a very promising inspection method of the qualities of agricultural products and foods [10-16]. This is because the imaging can reveal physical, chemical, geometrical, and optical parameters of the objects being inspected, whereas the spectroscopic method can provide the optical parameter only, because it can measure only the spectral intensity. However, the imaging has not been developed so far for the quantifying the degree of the infections. The separation between the fresh and infected fruits should be based on the quantification to prevent from discarding good ones with only insignificant amount of the infection. This is why the harvested citrus fruits are going through some forms of chemical treatments to protect the fruit skins from the pathogen infection [17-20]. In addition to the chemical treatments, there are several other ways of treating the fruit surfaces: Covering the surface with plant extracts and essential oils, and exposing to hot water and ultraviolet light [21]. However, these treatments are costly and can be harmful to people, especially the chemicals used for the chemical treatment. Besides, the fruits can also be damaged on the way of transporting them. This requires another separation method. This means that the surface treatment methods are convenient but costly and risky for health.

The spectral imaging is classified into three regimes such as multispectral, hyperspectral, and ultraspectral ones, depending on the transmitting spectral bandwidth of the spectral filter. When the filter can pass a spectral bandwidth of more than $10 \mathrm{~nm}$ or several different bandwidths, as in using color glasses, it is called multispectral imaging. When the passing bandwidths are near $1 \mathrm{~nm}$ to $10 \mathrm{~nm}$, and not more than $1 \mathrm{~nm}$, they are called hyper- and ultraspectral imaging, respectively. As the spectral bandwidth of the filter becomes smaller, the physical and chemical elements contained in the object to be tested can be identified with better accuracy because each individual element has its corresponding spectral wavelength. Among the imaging, the hyper- and multispectral imaging have been used very widely for estimating the quality of fruits, vegetables, meat, grains such as surface damage (bruises, chilling, and insect biting), surface quality (firmness, moisture content, hardness, rottenness, and fungi presence), biochemical components, and quality grades in the food and agricultural industries [22-25]. However, the multispectral imaging can be hardly used for the accurate detection of the green mold pathogen infection in the fruits, as it has a spectral range too wide to trace the presence of the pathogen, though the hyperspectral imaging was used to identify the pathogen presence in tangerine.

In this paper, a spectral intensity ratio and the behavior of spectral intensity changes in a hyperspectral imaging are used to estimate the degree of the green mold pathogen infection and to discriminate between fresh and infected lemons. The presence of green mold pathogens on the surfaces of lemons is identified with the use of the dominant wavelengths of the fresh lemon and the green mold pathogen colors, which are found in this paper.

\section{Materials and Methods}

The pathogen infection in the lemon is initiated by a hardly visible small damaged area in the skin of a lemon. The skin color of a healthy lemon is bright yellow when it is fully ripe [26]. However, the bright yellow skin color becomes greener as the green mold infection gets deeper. To show these color changes, the pathogen infected lemon samples are prepared and the hyperspectral images of each infected sample are taken and their intensity distributions are found in the following ways. 


\subsection{Pathogen Infected Lemon Samples Preparation and Changes in Skin Color}

A batch of the green mold pathogen infected lemon samples is prepared in the following way. Lemon samples, which are completely covered with the green mold pathogen, are prepared. Then, each of the completely pathogen infected lemon samples is put in the center of a chamber with 4 to 5 fresh lemons. This chamber is equipped with a thermometer and a humidifier, and the humidity and temperature is controlled to be kept as more than $80 \%$ and $25{ }^{\circ} \mathrm{C}$, respectively. The fresh lemon samples are having less than $2.2 \%$ intensity differences between them. The chamber is kept under such conditions for many days and for every $24 \mathrm{~h}$, then one of samples, which is not burst or unusually rotten, is selected and photographed with a camera with a spectral filter in front of its objective. 16 Hyperspectral filters in the spectral range of $500 \mathrm{~nm}$ to $650 \mathrm{~nm}$ are used. Each spectral filter has the spectral bandwidth of $10 \mathrm{~nm}$ centered at $500 \mathrm{~nm}$ to $650 \mathrm{~nm}$ for every $10 \mathrm{~nm}$ interval. The camera images of the lemon samples taken without spectral filters are shown in Figure 1 . There are 20 sample images of differently infected lemons. It is considered that the lemons are infected more as the sample number increases. Figure 1 clearly demonstrates that the bright yellow color of the fresh lemon skin turns more to green as the green mold infection becomes severe. The presence of the green mold pathogen is hardly recognized in images 1 to 7 , whereas its presence on the surfaces of the lemons is too obvious in samples numbered 8 to 20. The samples numbered 16 to 20 also show their morphology changes as the infection goes deeper.

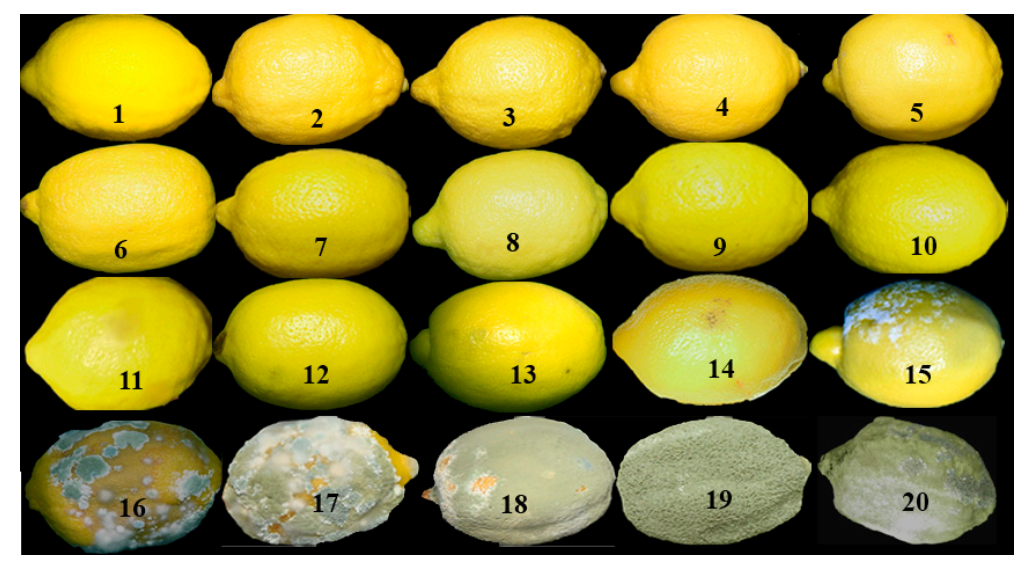

Figure 1. Lemon skin color changes with the increasing degree of the green mold pathogen infection. The lemons are more infected as the number increases: (1) healthy lemon, (2-19) infected lemons of different degrees and (20) lemon covered completely with the green mold pathogen.

The color changes can be quantitatively represented in histograms in 3-dimensional R (Red), G (Green), and B (Blue) color space. This is shown in Figure 2. The numbers represent 256 gray level. For the case of the undamaged lemon, i.e., fresh lemon as shown in the number 1 image of Figure 1 ; (1) $\mathrm{R}$ is the dominant color for the pixels with the gray levels up to 200, though the gray level difference between $\mathrm{R}$ and $\mathrm{G}$ is $\sim 30$, and (2) B component becomes distinct for the pixels with $\mathrm{R}$ and $\mathrm{B}$ gray levels greater than 200 but B gray level does not exceed 200. These facts indicate that the skin color of the lemon is mainly yellow but some gray colors are also there, although they are not very recognizable. The histogram as shown in Figure $2 b$ corresponds to the sample numbered 15 of Figure 1 . It shows that (1) $\mathrm{G}$ is the dominant color component but the gray level difference between $\mathrm{G}$ and $\mathrm{B}$ is not very large; (2) B component appears strongly for the pixels with R and G's gray level greater than 150, though the B's gray level does not exceed 200; and (3) only few pixels are colored by $\mathrm{R}$ and $\mathrm{G}$ with very low B component. These facts indicate that the color of the lemon skin shown in the sample numbered 15 of Figure 1 is shifted to the green side and more gray is added to it compared with the fresh lemon. This means that the lemon is now covered by green molds. Figure $2 \mathrm{c}$ is the RGB histogram of the image numbered 20 of Figure 1. It shows that (1) the gray levels of R, G, and B color components are almost 
the same to each other, though B population is much larger than (2) Figure $2 b$ and the highest gray level of $B$ is the same as those of R and G, and (3) a very few pixels have R and G's gray levels less than 100. These facts indicate that the skin color is changed mostly to the brighter gray. This means that the histogram can implicitly inform the presence of green and blue molds as demonstrated by the sample numbered 20 but cannot explicitly. As shown in Figure 2, the histograms of RGB color components for different stages of the infections are distinctively different from each other. The histogram in 3-dimensional color space informs implicitly the evolving stage of green and blue molds in lemon. However, it is difficult to define the pixel population difference of between different colors at each stage and between stages, which can serve as a criterion for segregating the not damaged lemons from the damaged ones. However, the spectral image, which will be shown in the next section, can provide the criterion.

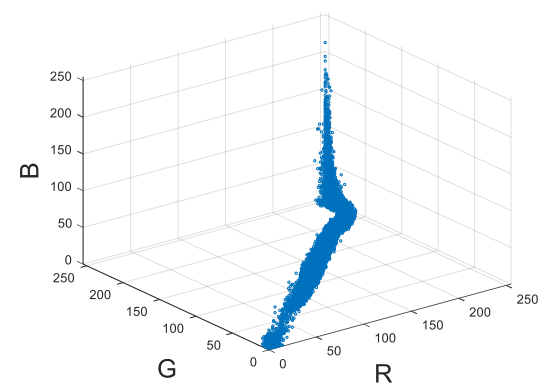

(a)

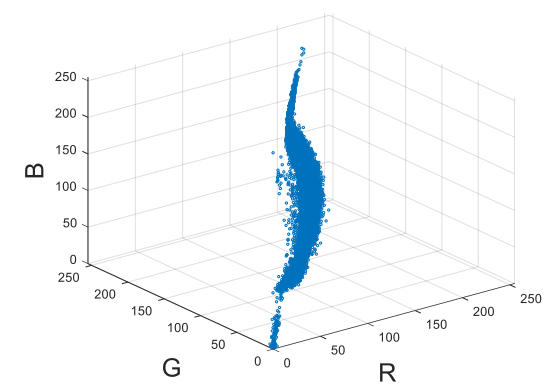

(b)

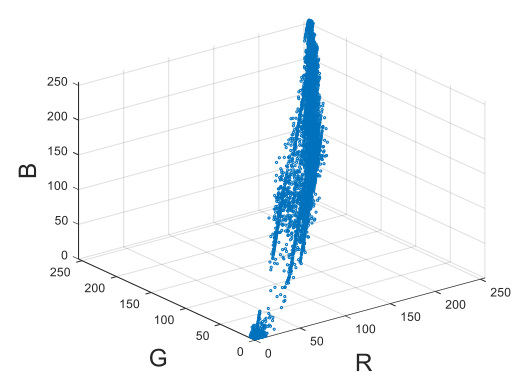

(c)

Figure 2. RGB histogram in 3-dimensional color space: (a) image 1 (b) image 15, and (c) image 20 of Figure 1.

\subsection{Experimental Set-Up for Taking Spectral Images and Their Intensity Distributions}

As earlier mentioned, each color of an object corresponds to a specific wavelength in near-UV (ultraviolet) to near-infrared wavelength regime. This means that if there is a spectral filter which passes only a narrow wavelength range within the above wavelength regime, it will be possible to take an image of the object, which has the specific color corresponding to the wavelength range. When a hyperspectral filter is represented by its center wavelength as in this paper, it indicates that the filter can transmit the light in the wavelength range of $\lambda_{k} \lambda_{k}-5 \mathrm{~nm}$ to $\lambda_{k}+5 \mathrm{~nm}$. In the spectral imaging, it is important to know that the spectral intensity distribution of the illumination source and the spectral transparency of the filter because the intensity distribution of each spectral image is linearly dependent on them.

Figure 3 shows the experimental set-up to take the hyperspectral images of lemons. It consists of a camera (2), a halogen lamp (4), two polarizers $(5,6)$, a power meter $(7)$ with a detector (8), and 16 hyperspectral filters in the spectral range of $500 \mathrm{~nm}$ to $650 \mathrm{~nm}$ for $10 \mathrm{~nm}$ interval (9). Nikon D810 with the detector resolution of $7360 \times 4912$ [27] is used as the camera. Each spectral filter has a diameter of $25 \mathrm{~mm}$ and a spectral bandwidth of $10 \mathrm{~nm}$, and is produced by Asahi spectra [28]. The transmittance 
of the filters in the spectral range of $500 \mathrm{~nm}$ to $650 \mathrm{~nm}$ is in the range of $79 \%$ to $80.5 \%$. The images with the spectral filters with less than $500 \mathrm{~nm}$ and greater than $650 \mathrm{~nm}$ have much lower brightness than those in $500 \mathrm{~nm}$ to $650 \mathrm{~nm}$. This is why they are not analyzed in this paper. Each spectral filter (9) is placed in front of the camera objective with its center matched on the camera optical axis. The objective is opened only through the filter. For each object, the spectral filters are changed 16 times. We used the data of the halogen lamp spectral power distribution [29] for calibrations of the optical system.

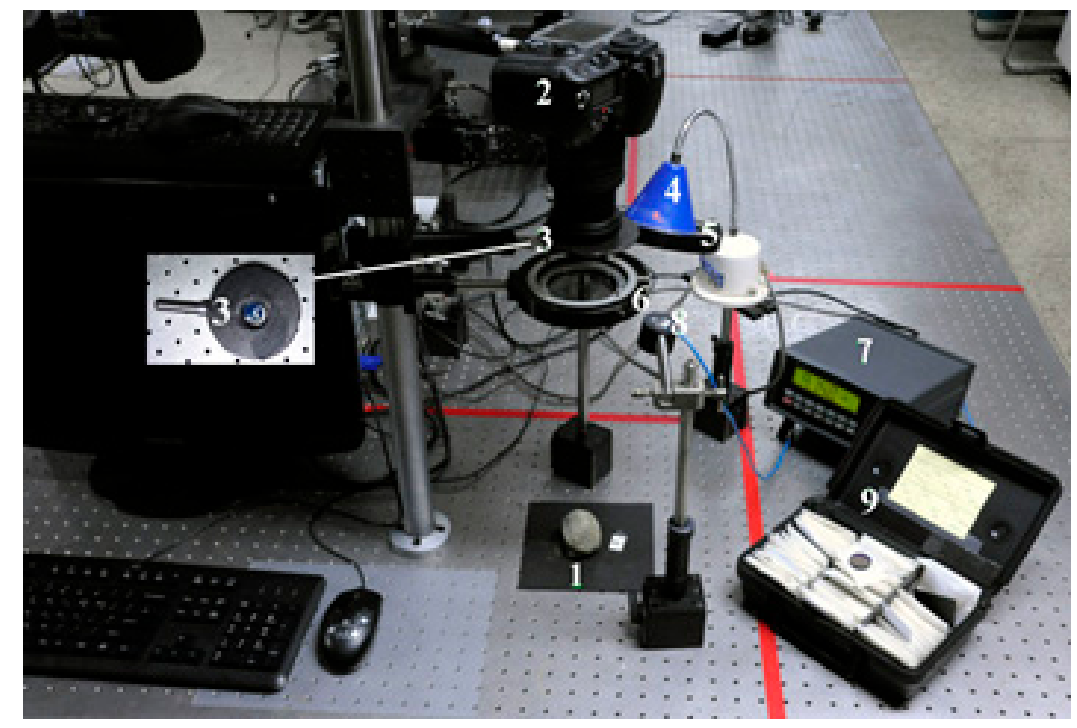

(a)

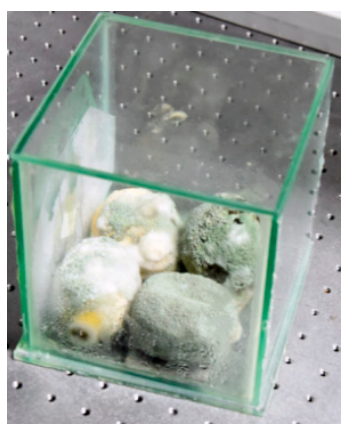

(b)

Figure 3. (a) Experimental set-up: (1) Object (Lemon), (2) Nikon Camera D810, (3) Spectral Filter, (4) a halogen lamp, (5) two polarizers, (6) analyzer, (7) a power meter, (8) a detector and (9) hyperspectral filters in the spectral range of $500 \mathrm{~nm}$ to $650 \mathrm{~nm}$ for $10 \mathrm{~nm}$ interval; (b) fully rotted lemons as source of green mold infection in special chamber.

Light intensity can be calibrated with the spectral power of the lamp for the lamp's spectral wavelengths. The accuracy of calibration can be in the range of $2 \%$ to $7 \%$ depending on the wavelength. The angle of the lamp beam to the normal direction of the optical table is $30^{\circ}$ (Figure 3 ). The illumination direction of the lamp is adjusted in such a way that the beam center is directed to the mid position of the object (1). Each of the two polarizers is located in front of the spectral filter. The filter is for taking only the surface reflected lights from the object [30]. Therefore the polarizer in front of the camera works as an analyzer. The object is at $\sim 350 \mathrm{~mm}$ distance from both polarizers. It is located so that its image appears at the mid area of the camera's viewfinder. The power meter is New Port 1830C [31], which has full-scale accuracy of $99.6 \%$. In this experiment, the illumination and photographing conditions are kept the same for each object. As described in Section 2.1, fully infected lemons are a source of green mold infection.

As the lemon shape is changing as the infected area grows, the objects' positions are carefully adjusted to be located at the mid-position of the viewfinder of the camera to keep the illumination condition the same for all cases. In the experiment, 16 objects with different degrees of infection are investigated. The objects can be 17 when the fresh lemon is included. For each object, 16 different spectral images are taken to observe the spectral property changes of each object's skin due to the pathogen infection. The spectral property is represented by the intensity average of each spectral image of the same object.

Figures 4-7 present the 16 spectral images of the objects shown in samples numbered 1, 12, 14, and 20 of Figure 1, respectively. In Figure 4a, the number in each spectral image represents the central wavelength $\lambda_{k}$ of the hyperspectral filter used. In images in Figures $4 \mathrm{~b}$ and 5, Figures 6 and 7, each 
lemon follows the spectral image orders as in Figure 4a. This means that the 1st row images are in the blue-green color region ( $500-530 \mathrm{~nm}$ ), the $2 \mathrm{nd}$ row-in the green color region ( $540-570 \mathrm{~nm}$ ), the $3 \mathrm{rd}$ row-in the yellow to orange color region (580-610 nm), and the 4th row-in the orange to red color region (620-650 nm) from left to right. Figure b of Figures 4-7 represents the grayscale version of the spectral images in the same order as the spectral images. When Figure a of Figures $4-7$ is converted into a 16-bit image in grayscale using the program OriginLab 2017, they are shown in Figures 8-11. The intensity value of each pixel of the image corresponds to the total light intensity passed through a spectral filter after reflecting from the lemon surface that is imaged to the pixel. The color scale shows the intensity level of each pixel. The gray scale images are intended to normalize the brightness of the spectral image because eye response to color brightness is different for different colors [32] These images inform that the fresh lemon, i.e., the leftmost image at 3rd row has the highest brightness at $580 \mathrm{~nm}$ and other yellow-orange images of the spectral range $590 \mathrm{~nm}$ to $610 \mathrm{~nm}$ look brighter than other spectral range images. Figure 5 distinguishes the yellow-orange colors. However, for the sample 12 as shown in Figure 6, the highest brightness still appears at $580 \mathrm{~nm}$. However, the images at the 2nd and the 3rd in the 2nd row, which correspond to $550 \mathrm{~nm}$ and $560 \mathrm{~nm}$, respectively, become brighter than other images in the 3rd row. Now the darkest images are in the 4th row. For the sample 14 as shown in Figure 7, the 2nd row images are brighter than other rows images and the 1st row images become brighter than those in Figure 6. The darkest images are still in the 4th row. For the case of Figure 7, the brightest images are the 2nd and 3rd ones in the 2nd row. The 1st row images look brighter than the images in the $3 \mathrm{rd}$ and 4 th rows. These spectral images indicate that the skin color of the lemon turns more green as the pathogen infected areas increase, and the green becomes the dominant color of the lemon, when the skin is completely covered by the pathogens. The blue-green colors are also more enhanced than yellow to red colors. Therefore, the spectral image clearly informs the presence of green and blue molds. Figures 5 and 6 clearly inform that the infected lemon can be fully characterized by the green colors, even the infected area is too small to be neglected. To see more clearly the brightness distribution of the skin surface and brightness changes in different spectral ranges due to the infection, the intensity distributions of the grayscale images in Figures 5-8 are plotted in a 3-dimensional space for the quantitative comparison between them.

Figures 8-11 represent the grayscale plotting of gray scale images in Figures 4-7, respectively, by considering lamp spectral intensity distribution, camera's spectral response, and the transparency of the spectral filters. The distribution represents only the lemon surface. As the lemons are having an oval shape, the intensity distribution over the lemon surface will not be uniform because of different heights.

The $x$ - and $y$-axes in Figures 8-11 correspond to images in columns and rows of Figures 4-7, respectively. The numbers $1-4$, which specify the scales of $y$-axis, represent the 1 st, $2 \mathrm{nd}, 3 \mathrm{rd}$, and 4th rows, respectively, whereas those of $x$-axis-the 1st, 2nd, 3rd, and 4th columns of Figures 4-7 respectively. Figures 8-11 allow quantitative comparison of different spectral images in differently affected lemons. The intensity distributions reflect mostly the surface shapes of the lemon. Figure 8 shows that (1) the intensity levels at $580 \mathrm{~nm}$ and $590 \mathrm{~nm}$ are higher than those at other spectral wavelengths, but the intensity distributions are more uniform over the image at $580 \mathrm{~nm}$ than at $590 \mathrm{~nm}$, (2) the areas having uniform intensity distribution are shrunk to the central region of the image for $590 \mathrm{~nm}$ and the uniformity is almost disappeared at $600 \mathrm{~nm}$ and $610 \mathrm{~nm}$, and (3) the spikes appearing at other spectral wavelengths are probably caused by the lemon's uneven surface. The light reflected from each surface area will be different from others. Figure 9 informs that (1) the spectral intensities of the spectral range above $570 \mathrm{~nm}$ are still dominant for other spectral ranges, but the areas are reduced compared with Figure 8, and (2) the intensity distributions of below $570 \mathrm{~nm}$ are gained more intensities over the large surface areas. This means that the green molds' encroaching the surface area is still going on.

Figure 10 reveals that (1) the intensity distribution of each spectral image in the spectral range of 540 to $570 \mathrm{~nm}$ is almost uniform and their intensities are close to the maximum gray level, (2) for the spectral ranges of 580 to $610 \mathrm{~nm}$, a large part of each image lost its spectral intensity if compared 
with Figure 9, but the central part of the image still keeps the maximum intensity level for $580 \mathrm{~nm}$; (3) for $500 \mathrm{~nm}$, the intensity distribution is almost uniform for the entire image area, except the spikes; and (4) the image lost much of its intensity for the spectral range greater than $610 \mathrm{~nm}$. This means that the green molds cover the entire surface of the lemon and blue molds are also growing in large parts of the lemon skin. Further, the color of the fresh lemon is much reduced. Figure 11 shows that (1) the intensity distributions of the spectral images in the spectral range smaller than $580 \mathrm{~nm}$ are very spiky for all their image areas, and their intensities are significantly higher than those of the images in spectral range greater than $570 \mathrm{~nm}$, and (2) the intensity distributions at $520 \mathrm{~nm}$ and $550 \mathrm{~nm}$ are higher than those of other spectral wavelengths. This means that there will be a significant amount of the blue mold along with the green mold pathogens and the lemon's skin is completely covered by both molds. The intensity spikes suggests that the molds have a hair shape.

Figures 8-11 inform that even in sample 14, a part of the lemon still retains its original color. This means that part of the lemon surface still remains untouched by the green and blue mold pathogens, at least for sample 14. Figures 8-11 enable comparison of absolute spectral intensities between different spectral images of differently infected lemons. However, the comparison is still difficult because they show intensity distributions of the images. To make the comparison easy, the intensity distribution of each spectral image at each infected state should be averaged and then normalized with the averaged spectral intensity distribution of the corresponding spectral image of the fresh/undamaged lemon. This will be shown in the next section.

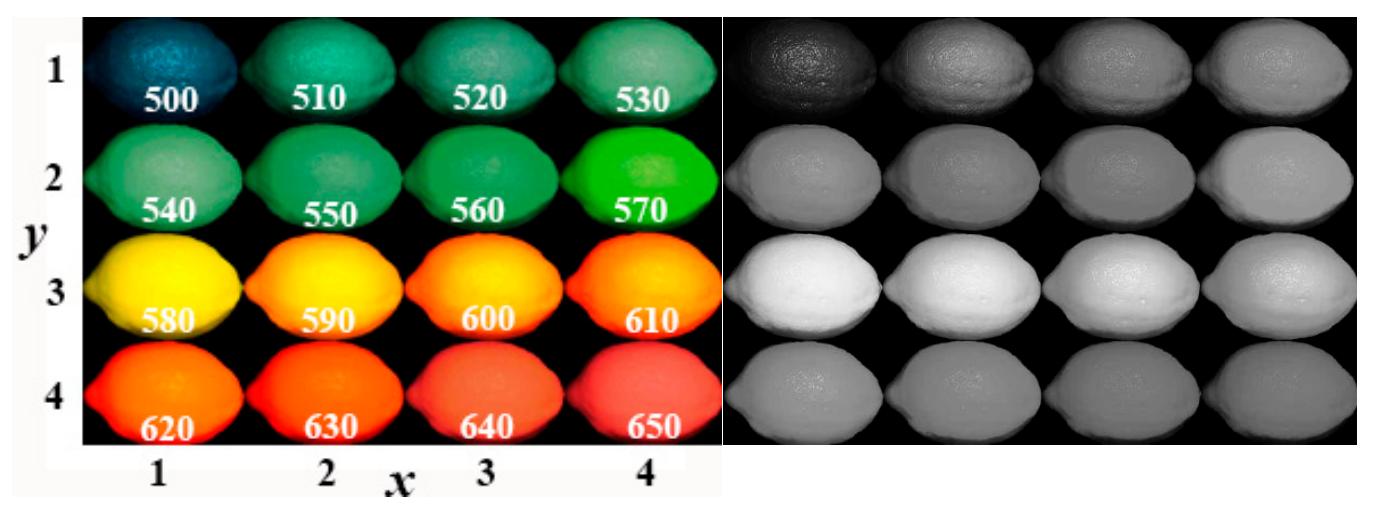

(a)

(b)

Figure 4. Sixteen spectral images of the lemon sample numbered 1 in Figure 1: (a) Spectral images from $500 \mathrm{~nm}$ at top left to $650 \mathrm{~nm}$ at bottom right in the order of left to right and top to bottom; (b) grayscale images of the spectral images in panel (a).



(a)

(b)

Figure 5. Sixteen spectral images of the sample lemon numbered 12 in Figure 1: (a) Spectral images from $500 \mathrm{~nm}$ at top left to $650 \mathrm{~nm}$ at bottom right in the order of left to right and top to bottom; (b) grayscale images of the spectral images in panel (a). 


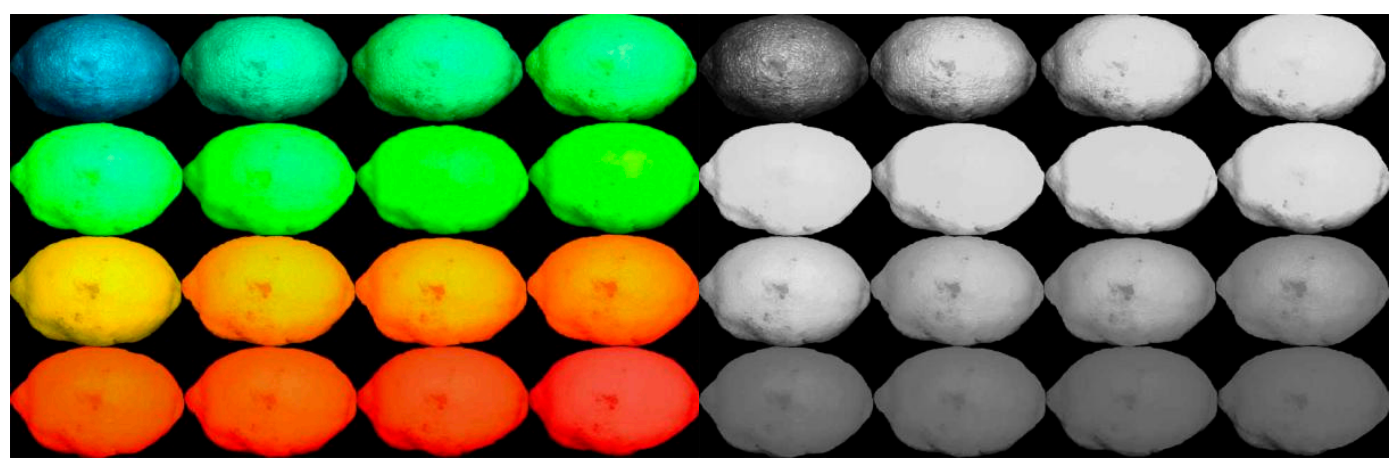

(a)

(b)

Figure 6. Sixteen spectral images of the sample lemon numbered 14 in Figure 1: (a) Spectral images from $500 \mathrm{~nm}$ at top left to $650 \mathrm{~nm}$ at bottom right in the order of left to right and top to bottom, (b) grayscale images of the spectral images in panel (a).

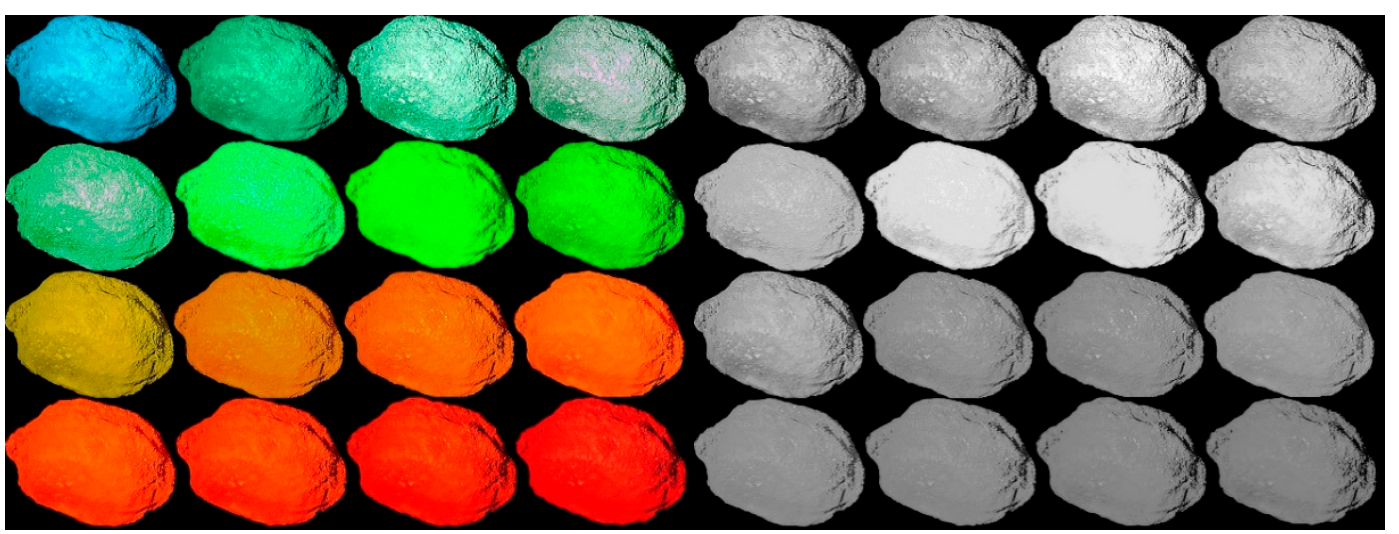

(a)

(b)

Figure 7. Sixteen spectral images of the sample lemon numbered 20 in Figure 1: (a) Spectral images from $500 \mathrm{~nm}$ at top left to $650 \mathrm{~nm}$ at bottom right in the order of left to right and top to bottom; (b) grayscale images of the spectral images in (a).



Figure 8. Intensity distribution of Figure 4 b. 


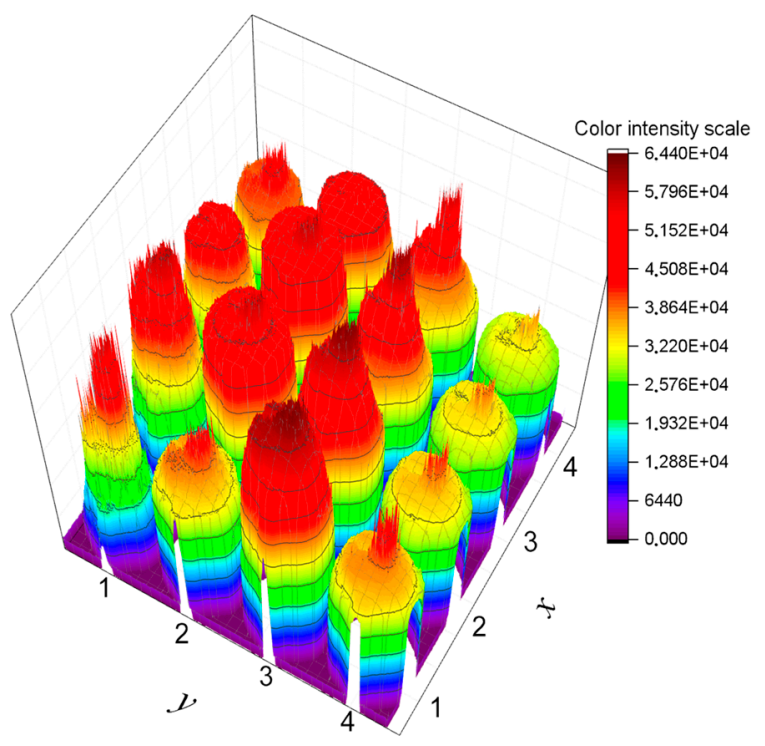

Figure 9. Intensity distribution of Figure 5b.

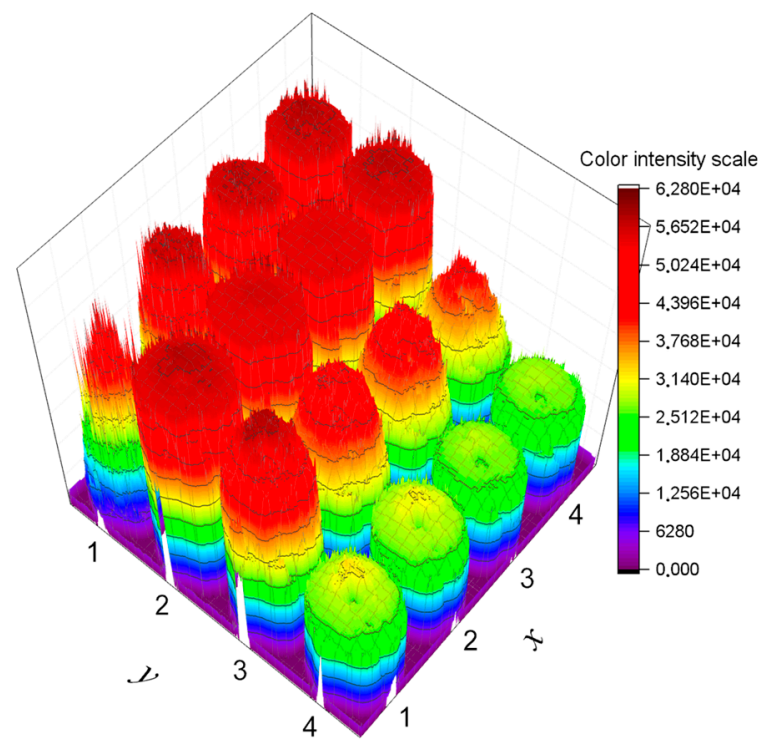

Figure 10. Intensity distribution of Figure 6b. 


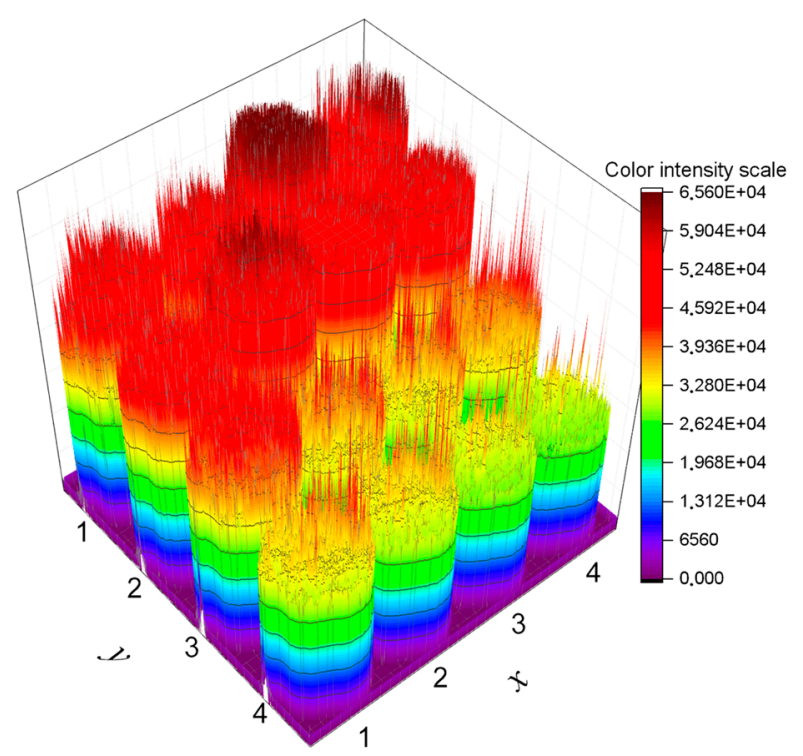

Figure 11. Intensity distribution of Figure $7 \mathrm{~b}$.

\section{Results and Discussion}

For the normalization, the intensity distribution of each spectral image from all infected states is averaged by adding up the intensities of all pixels making up the lemon image and then dividing it by the added number of pixels. These averaged intensities are divided by that of corresponding wavelength spectral image of the fresh lemon to normalize the intensities. This normalization eliminates the calibration problem incurred by the spectral intensity differences between different spectral ranges of the illumination lamp. The averaged spectral intensity $I_{\lambda_{k}}^{j}$ of $j$ th infected image at the spectral wavelength $\lambda_{k}$, where $j=1,2,3,4, \cdots, 17$, including the fresh lemon, $k=1,2,3,4, \cdots, 16$, can be expressed as

$$
I_{\lambda_{k}}^{j}=\frac{\sum_{i=1}^{n_{\lambda_{k}}^{j}} P_{i}}{n_{\lambda_{k}}^{j}}
$$

where $P_{i}$ and $n_{\lambda_{k}}^{j}$ represent $i$ th pixel intensity and the number of pixels making up each gray scale lemon image of $j$ th infected image at the spectral wavelength $\lambda_{k}$, respectively. The normalization can be expressed as

$$
\alpha_{\lambda_{k}}^{j}=\frac{I_{\lambda_{k}}^{j}}{I_{\lambda_{k}}^{0}}
$$

where, $\alpha_{\lambda_{k}}^{j}$ and $I_{\lambda_{k}}^{j}$ are the normalized coefficient and averaged intensity of $j$ th infected image at the spectral wavelength $\lambda_{k}$, respectively, and $I_{\lambda_{k}}^{0}$ the averaged intensity of not damaged lemon image at $\lambda_{k}$.

When the molds taken from the completely infected lemon, as in image 20 of Figure 1, are grafted on the skin surface of healthy lemons, the grafted area is hardly identified for the spectral filter of $540 \mathrm{~nm}, 550 \mathrm{~nm}$, and $560 \mathrm{~nm}$, as shown in Figure 12. Figure 12 presents photographic images of the grafted area taken with 16 spectral filters used for Figures 5-8. The numbers in this figure represent the central wavelengths of the spectral filters. The grafted area appears to be of a gray color with no filter as shown in the leftmost image of Figure 12. This means that the molds are probably having RGB color components with almost the same intensity. When the spectral filters are used, the molds are hardly identified in the spectral range of $540 \mathrm{~nm}$ to $560 \mathrm{~nm}$. This means that the spectral wavelength of the green molds is in the range of $540 \mathrm{~nm}$ to $560 \mathrm{~nm}$. For the case of red color, the molds are still 
identified up to $650 \mathrm{~nm}$. Therefore, a spectral filter in the range of $535 \mathrm{~nm}$ to $565 \mathrm{~nm}$ will be enough for the accurate detection of the green mold pathogen because the spectral range is the most sensitive to the pathogens.

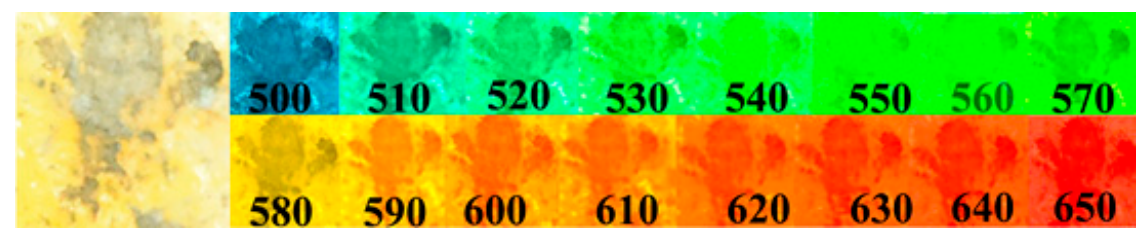

Figure 12. Photos of a lemon surface grafted with a green mold for the spectral range of 500 to $650 \mathrm{~nm}$. The 1st image is without filter.

The averaged spectral intensities of the 20 spectral images, as shown in Figure 1, are plotted in Figure 13 at each of the spectral wavelengths. The number of each curve corresponds to that of each sample in Figure 1. The intensity has the same relative scale as in Figures 8-11. Figure 13 shows that the spectral intensities at $570 \mathrm{~nm}$ have the minimum difference between samples.

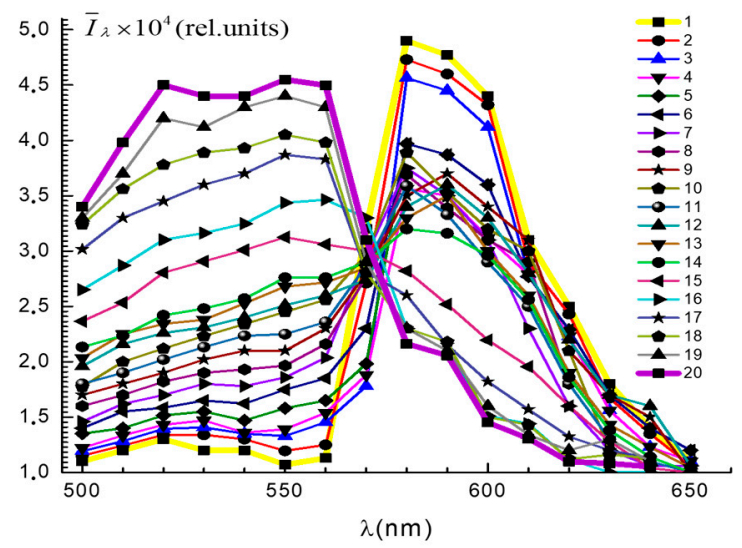

Figure 13. Spectral intensity distributions of 20 lemon samples shown in Figure 1, calculated with Equation (1).

This is expected because the dominant wavelengths of the green molds and the fresh lemon are in the range of $535 \mathrm{~nm}$ to $565 \mathrm{~nm}$ and $580 \mathrm{~nm}$, respectively. As sample 1 has the highest spectral intensity at $580 \mathrm{~nm}$ and very low spectral intensities at below $570 \mathrm{~nm}$, the spectral intensity at $570 \mathrm{~nm}$ has an intermediate value between these two values. Sample 2 behaves the same way as sample 1 . However, starting from sample 3, the intensity at $570 \mathrm{~nm}$ becomes lower than that of samples 1 and 2 and then increases as the sample number increase. For a few samples, the values can slightly exceed those of samples 1and 2. Along with this feature, Figure 13 also shows other features: (1) the fresh lemon has the lowest spectral intensity at $550 \mathrm{~nm}$, and (2) the intensity increases with sample numbers increase in the spectral ranges $510 \mathrm{~nm}$ to $560 \mathrm{~nm}$ though the increment between adjacent numbers is not the same. Also note that the spectral intensities at $550 \mathrm{~nm}$ and $560 \mathrm{~nm}$ are higher than those of the spectral range of $500 \mathrm{~nm}$ to $540 \mathrm{~nm}$ for most of the samples but for the samples 1 and 2, they are lower than those of $510 \mathrm{~nm}$ to $540 \mathrm{~nm}$. (3) The intensity decrease in the spectral ranges greater than $570 \mathrm{~nm}$ is not orderly because the intensities of other number samples are mixed together. (4) The spectral intensity is the smallest (largest) for the fresh (the completely covered with molds) in the spectral range of $500 \mathrm{~nm}$ to $560 \mathrm{~nm}$ and the largest (smallest) in the $570 \mathrm{~nm}$ to $650 \mathrm{~nm}$, except at $570 \mathrm{~nm}$. (5) The spectral intensity difference between samples numbered 15 and 20 is higher than that between 1 to 14. (6) The spectral intensities of samples 4 to 14 entangled themselves, i.e., the intensities no longer decrease with increasing sample numbers. (7) The spectral intensity of the fresh lemon has the highest intensity at $580 \mathrm{~nm}$ and the intensities decrease with increasing sample numbers for samples 1 to 3 , but they no 
longer decrease in corresponding to the increasing sample numbers, and the intensity at $590 \mathrm{~nm}$ is not too different from that at 580nm and shows the same behavior as the $580 \mathrm{~nm}$. (8) Samples 2 and 3 keep their spectral features as the same way as that of the sample 1, i.e., the fresh lemon, though the intensity ratio of sample 1:2:3 = 1.0:0.972:0.935, 1.0:0.971:0.943 and 1.0:0.985:0.938 for $580 \mathrm{~nm}, 590 \mathrm{~nm}$ and $600 \mathrm{~nm}$, respectively. (9) Samples 4-20 have spectral features completely different from those of sample 1 as manifested by the spectral intensity difference 0.5 between samples 1 to 3 and 4 to 20 at the spectral range $580 \mathrm{~nm}$ to $590 \mathrm{~nm}$. (10) The intensity difference at $600 \mathrm{~nm}$ is slightly smaller than that at $580 \mathrm{~nm}$ and $590 \mathrm{~nm}$ but the samples 1 to 3 are still distinctively separated from other samples. These features indicate that (1) the fresh lemon has the lowest spectral intensities at $550 \mathrm{~nm}$, but the intensities become higher as the green mold infection increases confirming again that the dominant spectral wavelength of the green mold is close to $550 \mathrm{~nm}$ and the lemons becomes more green due to the pathogen accumulations. The range is within $540 \mathrm{~nm}$ to $560 \mathrm{~nm}$ as demonstrated by Figure 12; (2) the spectral image can identify the infected states of the lemons, which are unrecognized with the naked eye; (3) the higher spectral intensities of the spectral range $550 \mathrm{~nm}$ insures that the spectral range is the most sensitive spectral range in quantifying the green mold pathogen infection in lemon and possibly for other citrus fruits too; and (4) the dominant spectral range of the fresh lemon is $580 \mathrm{~nm}$ to $590 \mathrm{~nm}$ and the samples 1-3 retain their spectral features but with other samples, their spectral intensity distributions in the spectral range exceeding $570 \mathrm{~nm}$ become completely different from those of samples 1 to 3, though the spectral intensities in the spectral range not exceeding $570 \mathrm{~nm}$ are slightly increasing as the sample number increases. The spectral intensity difference between samples 1 and 2 is less than 3\% for the spectral range $580 \mathrm{~nm}$ to $600 \mathrm{~nm}$, but this difference is slightly higher than the spectral intensity difference $2.2 \%$ between fresh lemons. This is why the spectral intensity of sample 2 at $570 \mathrm{~nm}$ is very similar to that of sample 1 . However, for samples 1 and 3, the differences are close to $7 \%$ for the spectral range $580 \mathrm{~nm}$ to $600 \mathrm{~nm}$. This difference is bigger than the spectral intensity difference $2.2 \%$ between fresh lemons. This informs that the pathogens are already on the skin surface of sample 3. This can be the reason why the spectral intensity of sample 3 at $570 \mathrm{~nm}$ becomes much lower than that of samples 1 and 2. This means that sample 2 can also be considered a fresh lemon.

Figure 13 shows that the normalized spectral intensity distribution defined by Equation (2) for the spectral range of $500 \mathrm{~nm}$ to $560 \mathrm{~nm}$ is derived as shown in Figure 14. In Figure 14, 19 curves are presented because the curve for the fresh lemon is used as the reference one. The numbers 1 to 19 correspond to the samples numbered 2 to 20, respectively. Figure 14 shows that (1) the difference between samples is more distinctive than that in Figure 13; (2) $\alpha$ values are increasing with increasing sample numbers for the specified spectral range and the normalized spectral intensity has the highest value at $550 \mathrm{~nm}$ for the samples numbered 9-20; (3) the $\alpha$ values of sample 2 and 3 at $550 \mathrm{~nm}$ are 1.15 and 1.3, respectively; and (4) among samples 2-14, sample 2 to 5 and 6 to 14 shows the highest $\alpha$ value differences for the spectral range $540 \mathrm{~nm}$ to $560 \mathrm{~nm}$. The difference is more than 0.15 . The coefficient $\alpha$ characterizes the degree of spectral characteristic changes in the lemon skin, i.e., the degree of pathogen infections on the lemon samples. The increasing $\alpha$ values with increasing lemon sample numbers indicate that the pathogens are growing more as the sample number increases. In Figure 14, the $\alpha$ value of the sample 20 at $550 \mathrm{~nm}$ is close to 4.2. This value represents the completely pathogen infected lemon. For the samples 17 to 20 , the $\alpha$ values are in the range of 3 to 4.2 . Therefore, the degree of pathogen infections on lemons can be quantified by calculating $\alpha$ values only at $550 \mathrm{~nm}$. According to Figure 13, the $\alpha$ value that can be considered as a fresh lemon will be up to 1.15 to $<1.3$. 




Wavelength

Figure 14. Normalized spectral intensity distribution of Figure 13 in the spectral range of 500 to $560 \mathrm{~nm}$.

The skins of lemons viewed with a microscope with the magnification of 200 are shown in Figure 15. Figure 15 compares the surface states of samples 1 and 6 . The fresh lemon surface specified by number 1 have no stains, i.e., it is clean but the sample 6 specified by number 2 is mottled by the pathogens. The thinner color areas represent the pathogen infected areas. When the infection goes deeper, all surfaces are densely covered by pathogens, as shown by sample 20 in Figure 1 . The $\alpha$ value of the sample 6 is 1.51 . However, it is noticed that the surface is greatly damaged, though it is hard to find the pathogen presence in sample 6 in Figure 1 with naked eye. Thus it can be said that it is not safe to use the lemon with $\alpha$ value of 1.51. It is considered that the $\alpha$ is useful to quantify the degree of pathogen infection on lemon skin.

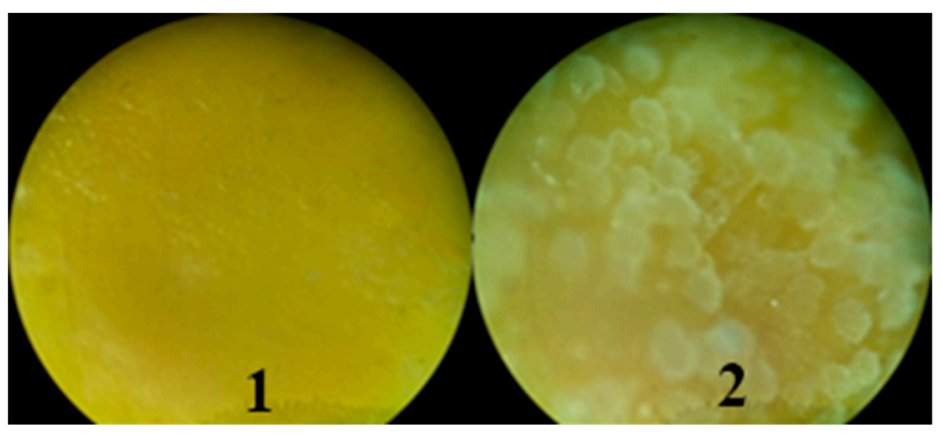

Figure 15. Microscope image of the surfaces with 200 times magnification: 1: Fresh lemon (Sample 1), and 2: Sample 6 with the $\alpha=1.51$.

\section{Conclusions}

Spectral imaging is not only a noninvasive, but also a very efficient method, of identifying and quantifying the lemons infected by green mold pathogens. The spectral range of $540 \mathrm{~nm}$ to $560 \mathrm{~nm}$ and $570 \mathrm{~nm}$ to $600 \mathrm{~nm}$ are good for identifying the states of lemon infected by the pathogens. The spectral range of $550 \mathrm{~nm}$ and $580 \mathrm{~nm}$, which is found to be the dominant wavelengths of the green mold pathogen and the lemon's skin color, is especially sensitive for quantifying the infection degrees, and discriminating between the fresh and infected lemons, respectively: the quantification can be done by calculating the normalized coefficient $\alpha$ at $550 \mathrm{~nm}$ and discriminating by spectral intensity variations at $570 \mathrm{~nm}$ and $580 \mathrm{~nm}$. The $\alpha$ value at which the lemon can be considered fresh will be less than 1.3.

The proposed method for determining the parameter $\alpha$ and calculating the spectral intensity variations can be useful in practice for detecting pathogens in other citrus fruits and foods too. Defining the $\alpha$ value range, which can help to discriminate the fresh and the infected lemons more accurately, and comparing this range with those for other citrus fruits will be the subject of our further research. 
The determined value range will enable the automatization of the process of inspecting the large amounts of citrus fruits in real-time.

Author Contributions: Y.V., Setting experimental setup and data collection; G.H., Total Planning and sample preparation; Y.K., Software design; T.V., Formatting, assisting manuscript preparation and English corrections; J.-Y.S., Full manuscript preparation and corresponding. All authors have read and agreed to the published version of the manuscript.

Funding: This work was supported by Priority Research Centers Program through the National Research Foundation of Korea (NRF) funded by the Ministry of Education (Grant Number: NRF-2018R1A6A1A03025542).

Conflicts of Interest: The authors declare that they have no conflict of interest.

\section{References}

1. Lakshmi, S.; Pandey, A.K.; Ravi, N.; Chauhan, O.P.; Gopalan, N.; Sharma, R.K. Non-destructive Quality Monitoring of Fresh Fruits and Vegetables. Def. Life Sci. J. 2017, 2, 103-110. [CrossRef]

2. Green Mold on Wood, Bread, Wall. This is How to Get Rid of This. Available online: https://healthida.com/ green-mold (accessed on 14 August 2019).

3. Bongomin, F.; Gago, S.; Oladele, R.; Denning, D. Global and Multi-National Prevalence of Fungal Diseases-Estimate Precision. J. Fungi. 2017, 3, 57. [CrossRef]

4. Chrdle, A.; Mallátová, N.; Vašáková, M.; Haber, J.; Denning, D.W. Burden of serious fungal infections in the Czech Republic. Mycoses 2015, 58, 6-14. [CrossRef] [PubMed]

5. Huh, K.; Ha, Y.E.; Denning, D.W.; Peck, K.R. Serious fungal infections in Korea. Eur. J. Clin. Microbiol. Infect. Dis. 2017, 36, 957-963. [CrossRef]

6. Ghosh, P.N.; Fisher, M.C.; Bates, K.A. Diagnosing emerging fungal threats: A one health perspective. Front. Genet. 2018, 9, 376. [CrossRef]

7. Garcia, M.D.; Chua, S.; Low, Y.; Lee, Y.; Francis, K.A.; Wang, J.; Nouwens, A.; Lonhienne, T.; Williams, C.M.; Fraser, J.A.; et al. Commercial AHAS-inhibiting herbicides are promising drug leads for the treatment of human fungal pathogenic infections. PNAS 2018, 115, E9649-E9658. [CrossRef]

8. Lluís, P. Penicillium digitatum, Penicillium italicum(Green Mold, Blue Mold). In Postharvest Decay. Control Strategies; Academic Press: Cambridge, MA, USA, 2014; chapter 2; pp. 45-102. [CrossRef]

9. Yahia, E.M. Postharvest Biology and Technology of Tropical and Subtropical Fruits; Woodhead Publishing: Cambridge, UK, 2011; pp. 437-516.

10. Su, W.; Sun, D. Multispectral Imaging for Plant Food Quality Analysis and Visualization. Compr. Rev. Food Sci. Food Saf. 2018, 17, 220-239. [CrossRef]

11. Adão, T.; Hruška, J.; Pádua, L.; Bessa, J.; Peres, E.; Morais, R.; Sousa, J.J. Hyperspectral Imaging: A Review on UAV-Based Sensors, Data Processing and Applications for Agriculture and Forestry. Remote Sens. 2017, 9, 1110. [CrossRef]

12. Report: Hyperspectral Imaging Market to Reach \$11.34 Billion in Five Years. Zion Market Research. 2017. Available online: https://www.zionmarketresearch.com/marketanalysis/hyper-spectral-imaging-systemmarket (accessed on 14 August 2019).

13. Gaurav, S.G. Hyperspectral Imaging: Technologies and Global Markets. BCC Research. 2016. Available online: https://www.bccresearch.com/marketresearch/instrumentationand-sensors/hyperspectral-imagingtech-markets-report-IAS106A.html (accessed on 14 August 2019).

14. Ahmed, M.R.; Yasmin, J.; Mo, C.; Lee, H.; Kim, M.S.; Hong, S.J.; Cho, B.K. Outdoor Applications of Hyperspectral Imaging Technology for Monitoring Agricultural Crops: A Review. J. Biosyst. Eng. 2016, 41 , 396-407. [CrossRef]

15. VetriDeepika, K.; Johnson, A.; LafrulHudha, M.; Ramya, M. Enhancement of Precision Agriculture Using Hyperspectral Imaging. IJTRD 2016, Special issue ETEIAC-16. 19-21.

16. Dale, L.M.; Thewis, A.; Boudry, C.; Rotar, I.; Dardenne, P.; Baeten, V.; Pierna, J.A.F. Hyperspectral Imaging Applications in Agriculture and Agro-Food Product Quality and Safety Control: A Review. J. Appl. Spectrosc. Rev. 2013, 48, 142-159. [CrossRef]

17. Mohammadi, P.; Tozlu, E.; Kotan, R.; Kotan Şenol, M. Potential of some bacteria for biological control of postharvest citrus green mold caused by Penicillium digitatum. Plant Protect. Sci. 2017, 53, 134-144. [CrossRef] 
18. El-Gali, Z.I. Control of Penicillium Digitatum on Orange Fruits with Calcium Chloride Dipping. J. Microbiol. Res. Rev. 2014, 2, 54-61.

19. Wang, W.; Liu, S.; Deng, L.; Ming, J.; Yao, S.; Zeng, K. Control of citrus post-harvest green molds, blue molds, and sour rot by the cecropin a-Melittin hybrid peptide BP21. Front. Microbiol. 2018, 9, 2455. [CrossRef]

20. Wang, W.; Deng, L.; Yao, S.; Zeng, K. Control of green and blue mold and sour rot in citrus fruits by the cationic antimicrobial peptide PAF56. Postharvest Biol. Technol. 2018, 136, 132-138. [CrossRef]

21. Papoutsis, K.; Mathioudakis, M.M.; Hasperué, J.H.; Ziogas, V. Non-chemical treatments for preventing the postharvest fungal rotting of citrus caused by Penicillium digitatum (green mold) and Penicillium italicum (blue mold). Trends Food Sci. Technol. 2019, 86, 479-491. [CrossRef]

22. Zhang, X.; Liu, F.; He, Y.; Li, X. Application of hyperspectral imaging and chemo metric calibrations for variety discrimination of maize seeds. Sensors 2012, 12, 17234-17246. [CrossRef]

23. Pu, Y.Y.; Feng, Y.Z.; Sun, D.-W. Recent progress of hyperspectral imaging on quality and safety inspection of fruits and vegetables: A review. Compr. Rev. Food Sci. Food Saf. 2015, 14, 176-188. [CrossRef]

24. El Masry, G.M.; Nakauchi, S. Image analysis operations applied to hyperspectral images for non-invasive sensing of food quality. A comprehensive review. J. Biosyst. Eng. 2016, 142, 53-82. [CrossRef]

25. Feng, C.; Makino, Y.; Oshita, S.; Francisco, J.; Martín, G. Hyperspectral imaging and multispectral imaging as the novel techniques for detecting defects in raw and processed meat products: Current state-of-the-art research advances. Food Control 2018, 84, 165-176. [CrossRef]

26. BBC Good Food. Available online: https://www.bbcgoodfood.com/glossary/lemon (accessed on 14 August 2019).

27. Digital Camera Nikon D810, User's Manual. Nikon Corporation USA. Available online: https://www.nikonusa.com/en/nikon-products/product/dslr-cameras/d810.html (accessed on 14 August 2019).

28. Asahi Spectra Inc. Available online: https://www.asahi-spectra.com (accessed on 14 August 2019).

29. MacDonald, L.; Giacometti, A.; Campagnolo, A.; Robson, S.; Weyrich, T.; Terras, M.; Gibson, A. Multispectral Imaging of Degraded Parchment. In International Workshop on Computational Color Imaging; Springer: Berlin/Heidelberg, Germany, 2013; pp. 143-157.

30. Son, J.Y.; Vashpanov, Y.; Jung, D.H.; Lee, D.S.; Kwack, K.D.; Kim, S.H. Polarized light for measuring a human skin feature indicating aging. Jpn. J. Appl. Phys. 2009, 48, 09LD06. [CrossRef]

31. Picowatt Digital Optical Power Meter Model 1830-C Newport. Available online: https://www.newport.com (accessed on 14 August 2019).

32. Valberg, A. Light Vision Color; John Wiley \& Sons Ltd.: Chichester, West Sussex, UK, 2005. 